\title{
Suppression of Adult Neurogenesis Impairs Olfactory Learning and Memory in an Adult Insect
}

\author{
Sophie Scotto-Lomassese, Colette Strambi, Alain Strambi, Aïcha Aouane, Roger Augier, Geneviève Rougon, and \\ Myriam Cayre \\ Laboratoire NMDA, Institut de Biologie du Développement de Marseille, Centre National de la Recherche Scientifique, Unité Mixte de Recherche 6156, \\ Campus de Luminy, 13288 Marseille Cedex 9, France
}

\begin{abstract}
Although adult neurogenesis has now been demonstrated in many different species, the functional role of newborn neurons still remains unclear. In the house cricket, a cluster of neuroblasts, located in the main associative center of the insect brain, keeps producing new interneurons throughout the animal's life. Here we address the functional significance of adult neurogenesis by specific suppression of neuroblast proliferation using $\gamma$ irradiation of the insect's head and by examining the impact on the insect's learning ability. Forty gray irradiation performed on the first day of adult life massively suppressed neuroblasts and their progeny without inducing any noticeable side effect. We developed a new operant conditioning paradigm especially designed for crickets: the "escape paradigm." Using olfactory cues, visual cues, or both, crickets had to choose between two holes, one allowing them to escape and the other leading to a trap. Crickets lacking adult neurogenesis exhibited delayed learning when olfactory cues alone were used. Furthermore, retention $24 \mathrm{hr}$ after conditioning was strongly impaired in irradiated crickets. By contrast, when visual cues instead of olfactory ones were provided, performance of irradiated insects was only slightly affected; when both olfactory and visual cues were present, their performance was not different from that of controls. From these results, it can be postulated that newborn neurons participate in the processing of olfactory information required for complex operant conditioning.
\end{abstract}

Key words: adult neurogenesis; mushroom body; neuroblasts; insect; house cricket; learning and memory; olfactory and visual conditioning; irradiation

\section{Introduction}

The dogma that the adult brain produces no new neurons has been overturned, but the question still remains of whether adult neurogenesis is a biologically relevant phenomenon. Observed in some invertebrates, as well as in all mammalian species examined so far, adult neurogenesis is conserved through evolution, and newly formed neurons are integrated in structures where information is processed and temporarily stored: the dentate gyrus of the hippocampus (Bayer et al., 1982; Gould et al., 1998; Eriksson et al., 1998) and the olfactory bulb (Corotto et al., 1993; Lois and Alvarez-Buylla, 1994) in vertebrates and the mushroom bodies in insects (Cayre et al., 1994, 1996a, Gu et al., 1999). Striking interrelations between neurogenesis and behavior have been reported in birds (Barnea and Nottebohm, 1996; Li et al., 2000). Likewise in rodents, factors that increase progenitor cell proliferation (running) or newborn neuron survival (enriched environment and learning) also improve learning performance (Kempermann et al., 1997; Gould et al., 1999a,b; Nilsson et al., 1999; van Praag et

Received June 6, 2003; revised Aug. 20, 2003; accepted Aug. 21, 2003.

This work was supported by the Fyssen Foundation. We thank Dr. P. Mouchy and Dr. C. Boyer for providing access to the irradiator, A. de Moya for building the escape maze, Dr. M. Giurfa for advice and enriching discussions, and F. Haigler for careful editing of this manuscript.

Correspondence should be addressed to Myriam Cayre, Laboratoire NMDA, Institut de Biologie du Développement de Marseille, Centre National de la Recherche Scientifique, Unité Mixte de Recherche 6156, Campus de Luminy , Case 907, 13288 Marseille Cedex 9, France. E-mail: cayre@ibdm.univ-mrs.fr.

Copyright $\odot 2003$ Society for Neuroscience $\quad$ 0270-6474/03/239289-08\$15.00/0 al., 1999; Rochefort et al., 2002). Conversely, glucocorticoids and stress, which inhibit neurogenesis, deteriorate learning and memory (Gould et al., 1997, 1998; Krugers et al., 1997; Lemaire et al., 2000). However, evidence of a direct causal link between the two phenomena is still scarce because of the difficulty of stopping neurogenesis in adults with high specificity. Using an antimitotic agent, Shors et al. (2001) showed that reduction of hippocampal neurogenesis impaired rat performance specifically in a hippocampus-dependent conditioning test. By contrast, impairment of neurogenesis by deletion of the presinilin- 1 gene did not cause a learning deficit, leading Feng et al. (2001) to suggest that new hippocampal neurons might be necessary to forget outdated information after cortical memory consolidation has occurred. In songbirds, destruction of adult-born neurons led to song deterioration with a recovery concomitant to compensatory neuronal production (Scharff et al., 2000). On the basis of these data, the precise role of adult neurogenesis in vertebrates still remains enigmatic.

No study has yet been devoted to the function of newborn neurons in invertebrates, although they constitute suitable and simple models. In insects, mushroom bodies are the main integrative centers of the brain because they receive multimodal sensory information (Mobbs, 1982, 1984; Gronenberg, 2001) and are involved in learning and memory processes (for review, see Heisenberg, 1994, 1998; Hammer and Menzel, 1995). In the house cricket Acheta domesticus, mushroom body neuroblasts are 
grouped in a cluster. By asymmetric cell division, the neuroblast self renews and forms a ganglion mother cell (GMC), which divides symmetrically to give rise to new mushroom body interneurons (Kenyon cells) throughout the insect's life (Cayre et al., 1994). As in vertebrates, both hormones (Cayre et al., 1994, 1997) and sensory stimuli (Scotto-Lomassese et al., 2000, 2002) regulate adult insect neurogenesis. To assess the putative participation of newly formed interneurons to learning processes, mushroom body neuroblasts were specifically destroyed by $\gamma$ irradiation, and learning abilities of control versus neuroblast-deprived animals were evaluated in a new learning paradigm, using olfactory conditioning, visual conditioning, or both.

\section{Materials and Methods}

\section{Animal treatments}

Adult females were isolated from the colony at the imaginal molt [day 0 (D0)], maintained under a 16/8 hr light/dark cycle in the rearing room, where the relative humidity was $55 \%$ and the temperature was $29 \pm 1{ }^{\circ} \mathrm{C}$. One group was used as a control, and the other was submitted to radioactive ${ }^{137} \mathrm{Cs} \gamma$ irradiation on D0. Crickets were cold-anesthetized and individually immobilized in a small assay tube. The tubes were then stuck on a $5 \mathrm{~cm}$ lead plate so that only the insect's head protruded and could be exposed to the $\gamma$ rays. The lead plate was placed in an irradiator (IBL 637; CIS Bio International) containing a source of ${ }^{137} \mathrm{Cs}$. To determine the optimal $\gamma$ ray dose, exposure ranging from 5 to 120 gray (Gy) was used, and neuroblast proliferation was examined either on D5 or D21 after irradiation.

\section{Estimation of cell proliferation and cell death after irradiation} 5-Bromo, 2'-deoxyuridine labeling. The thymidine analog 5-bromo, 2'deoxyuridine (BrdU), a marker of cell proliferation, was used on mushroom body spreadings as a fast method to evaluate the efficiency of irradiation on neuroblast destruction and to determine the optimal $\gamma$-ray dose.

Ten microliters of BrdU (40 mg/ml; Sigma, St. Louis, MO) were injected $2 \mathrm{hr}$ before insect dissection. Then the insect brain was dissected out; the neural sheath was removed; and each mushroom body cortex was excised and spread as a cell monolayer onto a polylysinated glass slide. Immunocytochemical detection of BrdU was then performed as described elsewhere (Scotto-Lomassese et al., 2000). BrdU-labeled cells in each spreading (i.e., per mushroom body) were counted under a microscope (Axiophot; Zeiss, Oberkochen, Germany).

Feulgen-Rossenbeck nuclear staining. Because the "spreading" technique does not preserve the integrity of the structure, we also performed histological serial sections of the brain followed by Feulgen-Rossenbeck nuclear staining, which allows visualization and counting of both pyknotic and mitotic cells.

Control and irradiated females were examined at the ages of 1,4 , and $7 \mathrm{~d}$. Cerebral ganglia were quickly dissected out in saline and then fixed for $6 \mathrm{hr}$ in Carnoy's fixative. After three $24 \mathrm{hr}$ washes in $95 \%$ ethanol and three $24 \mathrm{hr}$ washes in 1-butanol, tissues were embedded in paraffin and cut in $6 \mu \mathrm{m}$ serial sections. Sections were deparaffinized, rehydrated, and treated for DNA staining according to the method of Feulgen-Rossenbeck: DNA was hydrolyzed using $6 \mathrm{~N} \mathrm{HCl}$ for $1 \mathrm{hr}$ and stained with Schiff's reagent $(1 \mathrm{hr})$ at room temperature. Sections were counterstained in $0.4 \%$ picro-indigo-carmin. Mitotic cells and pyknotic nuclei were counted on serial sections of mushroom bodies. To correct the overestimation linked to counting the same nucleus on two adjacent sections, the following formula (Abercrombie, 1946) was applied: $N=$ $[(n \times t) /(d+t)]$, where $t$ was section thickness; $d$, nucleus diameter; and $n$, number of counted nuclei.

\section{Data acquisition for ovipositor movements and locomotor} activity recordings

Seven days after irradiation (D7), ovipositor movements and locomotor activity were continuously detected by radio tracking (Fig. 1) over 8 consecutive days. A ${ }^{192}$ Ir wire (1 mm length, $0.3 \mathrm{~mm}$ diameter, $1.5-2$ $\mathrm{MBq}$ activity) was glued to the tip of the female cricket's ovipositor (Rage

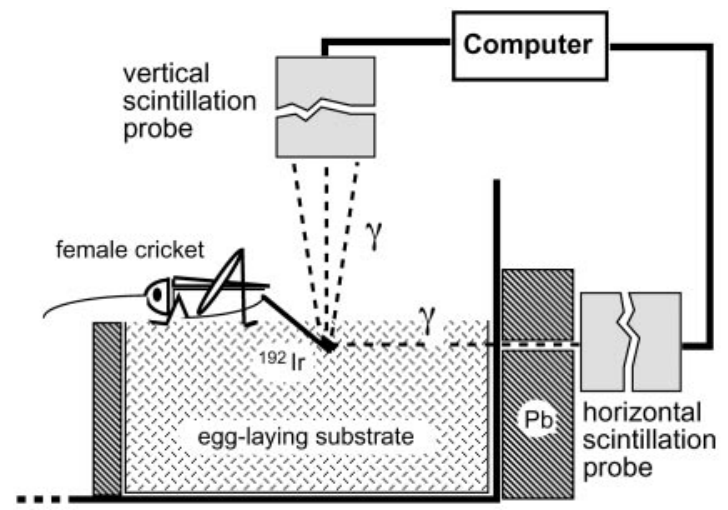

Figure 1. Experimental device used to record and quantify locomotor activity and oviposition movements (adapted with permission from Cayre et al., 1996b). The horizontal probe received $\gamma$-rays only when the tip of the ovipositor was inserted into the egg-laying substrate. The vertical probe received levels of radiation inversely proportional to its distance from the source. Pb, Lead wall; ${ }^{192} \mathrm{Ir}$, iridium wire that emits $\gamma$-rays.

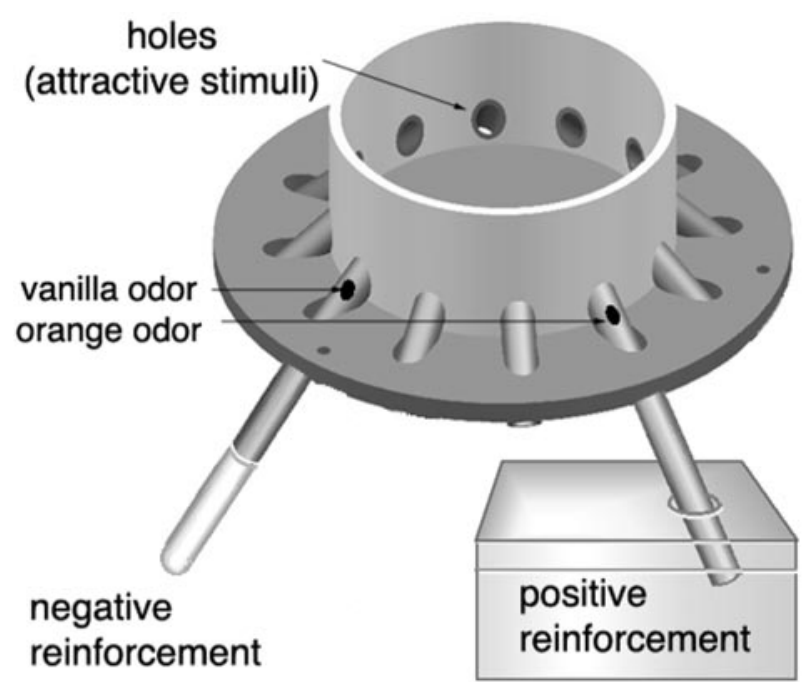

Figure 2. Experimental device of the escape paradigm used to evaluate the learning and retention abilities of control and irradiated crickets. The cricket, placed in the center of the arena, has to choose between two open holes to find the one that will allow it to escape into a large cage. Olfactory cues, visual cues, or both are proposed. Here, only olfactory cues are represented. The legs of the arena have not been represented for clarity.

and Renucci, 1985), and the $\gamma$-rays emitted by the radio element were detected and converted to electrical impulses by two scintillation probes (SMIG/E 724; Nardeux) recording locomotor activity or ovipositor movements, respectively (Renucci et al., 1992). The scintillation probes were interfaced with a personal computer (IPC), which stored the data at fixed $6 \mathrm{sec}$ intervals. Data were then pooled in $30 \mathrm{sec}$ sequences.

\section{Escape paradigm}

The device was a circular arena, the wall of which was perforated by 12 equally spaced holes allowing the cricket to escape (Fig. 2). For the present study, only two holes were opened, one leading to a reward, a large dim cage with food and water, and the other leading to a punishment, a small tube where animals were trapped. Olfactory cues, visual cues, or both were available to enable the animal to differentiate the two holes. Filter papers impregnated with odorant cues were stuck inside the tubes leading either to the escape cage or to the trap, $7 \mathrm{~cm}$ away from the hole in the arena wall (Fig. 2). Orange odor (Pranarom-natessence, 1:500) was associated with the reward hole, and vanilla odor (natural food extract of vanilla) was associated with the punishment hole. These associations were chosen because crickets usually exhibit a spontaneous 
preference for vanilla over orange. They were thus forced to reverse their initial preference. Associating a preferred odor with the punishment and a less attractive odor with the reward strengthens the significance of the animal's choice on the basis of learning rather than at random. Visual cues were rectangles $(5.5 \times 5 \mathrm{~cm})$, which were black, white, or striped, glued on the gray wall of the arena right above the holes. To rule out the possibility that other visual stimuli outside the arena could affect the animal's behavior, the arena was rotated by $45^{\circ}$ between each trial, and experiments were performed in a dark room with a strong light immediately above the apparatus, specifically illuminating the arena.

Two days before the beginning of the experiment (D7), controls and irradiated animals were deprived of food. The initial spontaneous preferences of crickets for orange or vanilla were tested $4 \mathrm{~d}$ before the beginning of the training session using olfactory cues, in two sessions of $10 \mathrm{~min}$ each according to the protocol of Matsumoto and Mizunami (2000), and the very few crickets that exhibited an initial preference for orange over vanilla were discarded.

One training session was performed each day and consisted of 10 trials. In the first two trials, only one hole was opened (leading randomly to punishment or to reward); therefore, we made sure that the insect successively visited the two holes. In the next eight trials, the two holes were opened, and the crickets had to choose between them. Usually, crickets spent a few minutes exploring the arena before choosing a hole ( $1 \mathrm{~min} 24$ $\mathrm{sec} \pm 11 \mathrm{sec}$ for control crickets vs $1 \mathrm{~min} 46 \mathrm{sec} \pm 13 \mathrm{sec}$ for irradiated crickets; $p=0.196$ ); insects that remained in the arena $>10$ min without entering any hole were discarded from the experiment (in total, only two control and four irradiated females had thus been discarded). Punishment and reward lasted $8 \mathrm{~min}$ each. The daily training session was repeated (with a maximum of $5 \mathrm{~d}$ ) until the animal's score reached 7 of 8 or 8 of 8 , indicating that the task had been learned.

Two retention sessions were performed $24 \mathrm{hr}$ and $7 \mathrm{~d}$ after the last learning session and consisted of one choice trial without reward or punishment so that reactivation of learning could be excluded. For each trial, we calculated the retention index (RI), which is the number of successful animals minus the number of unsuccessful animals divided by the total number of crickets. Therefore, an RI of 1 means that all animals chose the correct hole; a null RI means that half the animals tested were successful and half were wrong; and an RI of -1 means that all animals chose the wrong hole (i.e., the hole leading to the trap).

\section{Data analysis}

The number of BrdU-labeled cells was expressed as mean \pm SEM per brain, and the significance of differences was evaluated using the nonparametric Mann-Whitney $U$ test. For behavioral analysis, Cox's test of survival and a variant analysis ( $t$ test) were chosen to compare the rate of acquisition and the retention abilities between control and irradiated animals.

\section{Results}

\section{Determination of the optimum dose of $\gamma$ irradiation}

The effect of irradiation on neuroblast proliferation was examined 5 and $21 \mathrm{~d}$ after exposure to the cesium source. Whatever the delay, irradiation induced a dose-dependent reduction in the number of BrdU labeled neuroblasts and ganglion mother cells. As shown in Figure 3, the values draw asymptote-shaped curves, pointing out that $40 \mathrm{~Gy}$ was the lowest $\gamma$-ray dose allowing a prominent effect. At this dose, only $10 \%$ of the neuroblasts kept proliferating after $5 \mathrm{~d}$, and these values dropped to only $1 \% 3$ weeks after irradiation. Consequently, the dose of $40 \mathrm{~Gy}$ was chosen for all the following experiments.

Furthermore, to check a posteriori the efficiency of irradiation for each irradiated insect subjected to the "escape paradigm," all the insects used in behavioral studies were injected with BrdU on day 4 , and at the end of the experiment, they were killed, and the number of BrdU-labeled cells in their mushroom bodies was counted. We observed that all irradiated crickets used in the behavioral experiments exhibited a strong decrease in neuroblast

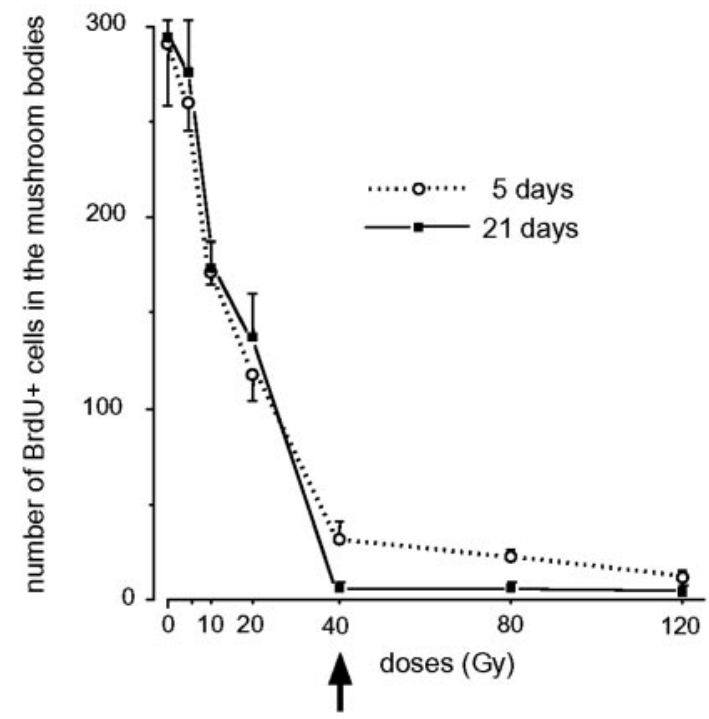

Figure 3. Dose-response effect of $\gamma$ irradiation with ${ }^{137} \mathrm{Cs}$ on neuroblast proliferation. Irradiation was performed on the first day of adult life (D0). BrdU was injected either on D5 (solid line) or on D21 (dotted line), and crickets were killed $2 \mathrm{hr}$ after BrdU injection. Error bars indicate SEM. At $5 \mathrm{~d}, n=6,6,6,3,5,8$, and 6, respectively, for increasing doses; at $21 \mathrm{~d}, n=6,2,3,6$, 6,4 , and 3 , respectively, for increasing doses. The arrow indicates that the dose of 40 Gy was chosen for the following experiments.

proliferation $(22.3 \pm 5.8 \mathrm{BrdU}$-labeled cells in irradiated insects vs $718.9 \pm 169.4$ cells in control animals).

\section{Effects of irradiation on mushroom body cells}

After Feulgen-Rossenbeck staining, mitotic cells were easily recognizable by their condensed chromatin, showing metaphase or anaphase figures, whereas small, dense nuclei characterized degenerating cells (Fig. 4). Neuroblasts are grouped in a cluster of cells localized at the apex of the mushroom body cortex (Fig. 4A); their nucleus is twice as big as that of differentiated Kenyon cells. GMCs lie immediately beneath the neuroblasts and are of intermediate size (Cayre et al., 1994). Newly born Kenyon cells fill the most central part of the cortex, whereas older mature interneurons are located more laterally (Cayre et al., 1996a; Malaterre et al., 2002).

In control brains, a few pyknotic nuclei were randomly scattered among the mature Kenyon cells $(6.3 \pm 1.7,4.2 \pm 2.0$, and $4.9 \pm 1.8$ at days 1,4 , and 7, respectively; $n=3,6$, and 5). By contrast, $1 \mathrm{~d}$ after 40 Gy irradiation, although only a few degenerating neuroblasts could be seen, numerous pyknotic nuclei $(197.4 \pm 35.0 ; n=5)$, corresponding to GMCs and newly generated interneurons, were observed at the bottom of the proliferative area (Fig. 4B). Only rare pyknotic figures were found among mature Kenyon cells. Four days after irradiation, the proliferative area looked disorganized, and pyknotic figures ( $40.4 \pm 7.5$ nuclei; $n=5$ ) corresponded to neuroblasts (Fig. $4 C$ ). Seven days after irradiation, the number of pyknotic cells, mostly neuroblasts, decreased $(19.5 \pm 6.6 ; n=4)$. No difference in the number of pyknotic mature interneurons could be demonstrated between irradiated and control crickets. The mitotic index, defined as the number of mitotic figures observed in the proliferative area 1-7 d after irradiation, was strongly and significantly reduced compared with that of controls (day $1,0.2 \pm 0.2$ vs $32.5 \pm 2.2 ; p<$ 0.0001 ; day $2,2.2 \pm 0.2$ vs $29.5 \pm 1.8 ; p<0.002$; day $7,0.3 \pm 0.3$ vs $23.0 \pm 2.5 ; p<0.008)$. These results emphasize that although 

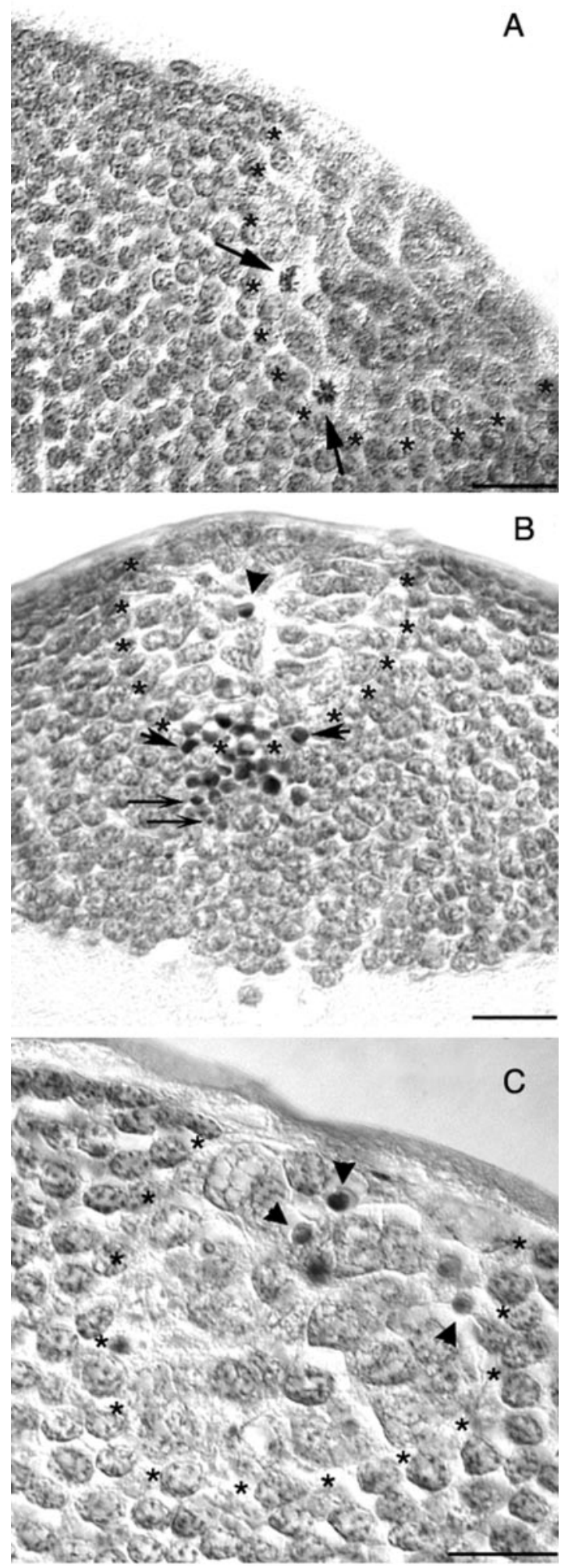

Figure 4. Effect of $40 \mathrm{~Gy} \gamma$-irradiation on mushroom body cells. Histological sections of the mushroom body cortex stained with the nuclear coloration of Feulgen-Rossenbeck are shown. The proliferative area is delineated by black asterisks. Irradiation was performed on the first day of adult life (DO). A, Before irradiation. Arrows indicate dividing neuroblasts in M-phase. B, One day after irradiation. The arrowhead indicates pyknotic nuclei of one neuroblast; small arrows point to pyknotic ganglion mother cells; and long arrows indicate pyknotic immature neurons. C, Four days after irradiation. Numerous neuroblasts exhibit pyknotic morphology, indicating their degenerescence (arrowheads). No mitotic figures can be observed. Scale bars, $20 \mu \mathrm{m}$.
40 Gy $\gamma$-ray irradiation was very efficient in killing proliferating cells or immature neurons, it did not induce apoptosis of differentiated interneurons.

\section{Evaluation of the general effect of irradiation}

Ovarian maturation in young adult females requires extensive cell proliferation. We thus explored the effect of irradiation on ovarian maturation by measuring the length of the longest distal oocytes and by checking the presence of vitellogenic mature oocytes in the abdomen. In both control and irradiated crickets, ovaries appeared to be fully developed, with $>10$ vitellogenic chorionated, free oocytes in the abdominal cavity. The longest nonchorionated distal oocytes exceeded $850 \mu \mathrm{m}$ in all cases. Therefore, no significant difference could be observed between the two experimental groups. This suggests that the lead plate used to protect the insect body was highly efficient; therefore, only the insect head was exposed to noxious irradiation.

Moreover, neither locomotor activity (1011 \pm 99.9 movements/d for control females vs $930 \pm 137.1$ for irradiated ones; $p=0.64$ ), used as an index of good health, nor the innate egglaying behavior ( $83.62 \pm 58.44$ oviposition sequences/d for control females vs $50.35 \pm 30.05$ for irradiated ones; $p=0.63$ ) were altered in irradiated animals.

In addition, we checked that irradiated crickets were still able to smell and discriminate between orange and vanilla, the two odors used as olfactory cues in our associative learning paradigm. Using the odor preference test described by Matsumoto and Mizunami (2000), we observed that irradiated crickets spent more time on the vanilla than on the orange source, indicating a clear preference for vanilla over orange, as already observed in control crickets $(93 \pm 3 \%$ of the time spent on an odor source was spent on vanilla for control crickets vs $92 \pm 8 \%$ for irradiated crickets).

Thus, as with a 40 Gy dose, the general physiology of the irradiated females was not affected; irradiation appears to be a method compatible with behavioral studies.

\section{Effects of irradiation on learning performance in the escape paradigm}

Olfactory cues

As previously quoted, a majority of both control and irradiated crickets exhibited a stronger preference for vanilla than for orange. The few animals that presented a spontaneous preference for orange were not used in the escape paradigm because the orange odor was associated with the reward, and the vanilla odor was associated with the punishment.

Compared with control animals, irradiation induced a significant impairment of learning abilities when crickets were provided with olfactory cues only $(p=0.016)$. As shown in Figure $5 A$, acquisition was delayed in irradiated animals compared with control ones. As early as the first session, 35\% of control animals were successful, whereas none of the irradiated females succeeded ( $p=0.024)$. On day $2,76 \%$ of control crickets had learned the task versus only $15 \%$ of irradiated ones $(p=0.003)$. After four sessions, although the task was mastered by $94 \%$ of control animals, only $77 \%$ of irradiated animals succeeded.

\section{Visual cues}

Acquisition in control animals was slower than for olfactory discrimination, but $100 \%$ of them learned the task in four sessions (Fig. 5B). Conversely, irradiated animals learned faster with visual than with olfactory cues. Therefore, there was no significantdifference in the time course of learning between control and irradiated crickets provided with visual cues alone $(p=0.098)$. 
A

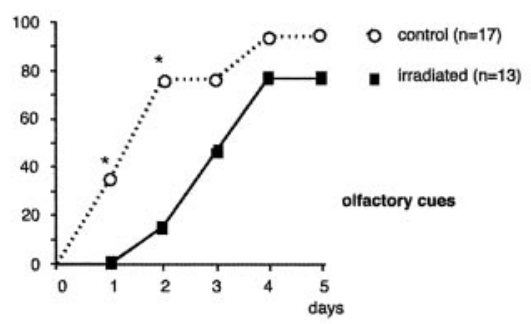

B
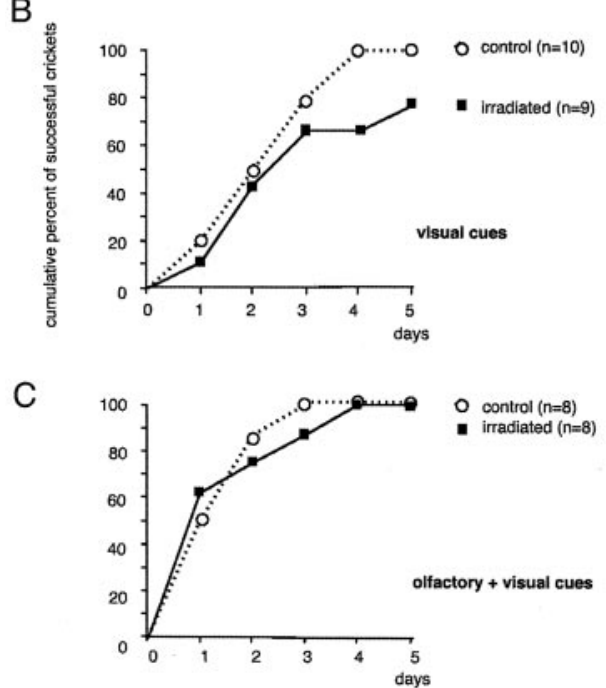

D

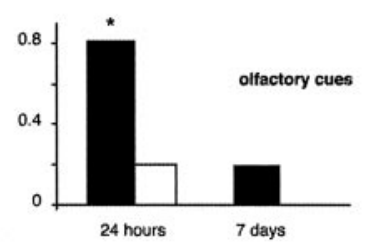

$E$

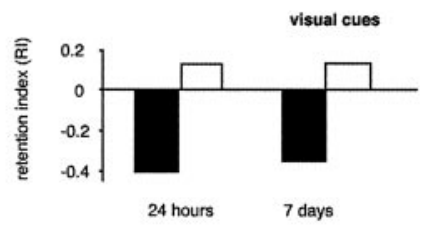

$\mathrm{F}$

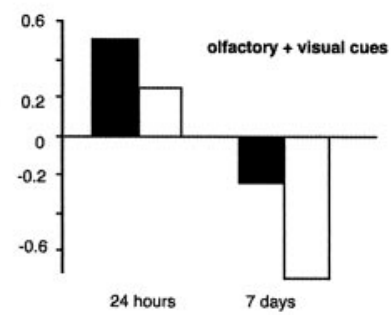

Figure 5. Effect of irradiation on learning and retention performance of crickets in the escape paradigm when olfactory $(A, D)$, visual $(B, E)$, or both olfactory and visual $(C, F)$ cues were provided. Irradiation was performed on the first day of adult life (DO); behavioral study started $7 \mathrm{~d}$ after irradiation. Retention tests were performed 1 and $7 \mathrm{~d}$ after the last learning session. The Rl is the number of successful animals minus number of unsuccessful animals divided by total number of animals tested. *Significant difference ( $p<0.05$ ) between the two experimental groups (control vs irradiated).

However, whereas all control females were successful after five training sessions, $22 \%$ of irradiated females never mastered the task properly.

\section{Olfactory and visual cues}

When the animals were provided with both olfactory and visual cues, acquisition was very fast, and $100 \%$ of the animals learned the task in three or four training sessions (Fig. $5 C$ ). The performances for the two groups were thus better than for visual or olfactory cues alone. No difference between control and irradiated groups was observed either in the speed of acquisition or in the number of successful insects $(p=0.371)$.

Our results suggest that the destruction of mushroom body neuroblasts by irradiation affects learning performance of the insects specifically when the conditioning relies on olfactory cues.

\section{Effects of irradiation on retention performance \\ Olfactory cues}

Twenty-four hours after the end of acquisition, control crickets presented a high retention score (RI, 0.82; Fig. $5 D$ ). By contrast, the RI of irradiated crickets was greatly reduced (RI, $0.2 ; p=$ $0.05)$. Seven days after learning, the RI of irradiated females was null, indicating a random choice of the holes by the animals. Control crickets presented a positive but low RI, suggesting that retention of the conditioning was weak after this time lapse.

To check whether irradiation disturbed the mature Kenyon cell networks, successful control animals, which were provided with olfactory cues, were exposed to $\gamma$-rays $6 \mathrm{hr}$ after the last learning session. Their retention ability, evaluated $24 \mathrm{hr}$ later, was not disturbed (Fig. 6) because these postlearning irradiated animals remembered the task almost as well as control animals (RI, $0.64)$. This observation suggests that irradiation did not affect the neural networks involved in the formation of the memory. By contrast, suppressing neuron production by irradiation on $\mathrm{D} 0$ produced adult crickets with retention deficiencies in our olfactory conditioning.

Visual cues

Although crickets were able to learn the visual task, as early as $24 \mathrm{hr}$ after training, neither irradiated nor control animals could perform it correctly (Fig. 5E). This performance was not improved after $7 \mathrm{~d}$.

Olfactory and visual cues

When both visual and olfactory cues were present during conditioning, the RI at 24 hr after learning was twice as high in the control group as in the irradiated group (Fig. 5F). However, $7 \mathrm{~d}$ after the last training session, the RI of control crickets was slightly negative, suggesting that they could not remember the task properly. Interestingly, in irradiated crickets, the RIs even reached highly negative scores, indicating that most of them chose the odor they initially preferred.

\section{Discussion}

To examine the functional significance of adult neurogenesis in the adult cricket brain, we developed a method for selectively killing mushroom body neuroblasts and examined the behavioral performance of crickets lacking adult neurogenesis. Our results show for the first time that newly generated neurons improve learning speed in operant olfactory conditioning.

Hydroxyurea, an antimitotic drug commonly used to prevent mushroom body formation in larval Drosophila and honeybees (de Belle and Heisenberg, 1994; Malun, 1998), failed to give satisfactory results in adult crickets because doses necessary to obtain robust reduction of neurogenesis presented toxic effects (M. Cayre, unpublished results). We therefore searched for a more selective way to destroy neuroblasts. Taking advantage of the greater sensitivity of proliferating cells to ionizing irradiation compared with postmitotic cells, we performed insect head irradiation to kill progenitor cells. Progenitor cells of the hippocampus and subventricular zone may be destroyed by irradiation (Peißner et al., 1999; Tada et al., 1999, 2000). Irradiation had previously been used successfully during the development of the moth Manduca sexta to reduce the population of glial cells in the antennal lobe (Oland et al., 1988). Apart from a few dividing cells present close to the medulla of the optic lobe, previous extensive BrdU labeling of the adult cricket brain demonstrated that the mushroom body cortex was the main brain structure where neural progenitors persisted (Cayre et al., 1996a). Our results clearly demonstrated the efficiency of $\gamma$-ray exposure because a dose of $40 \mathrm{~Gy}$ was necessary and sufficient to trigger an irreversible suppression of cell proliferation. In irradiated crickets, pyknotic figures were restricted to neuroblasts and to newly generated cells; mature Kenyon cells were not affected by $40 \mathrm{~Gy}$ irradiation. Fur- 

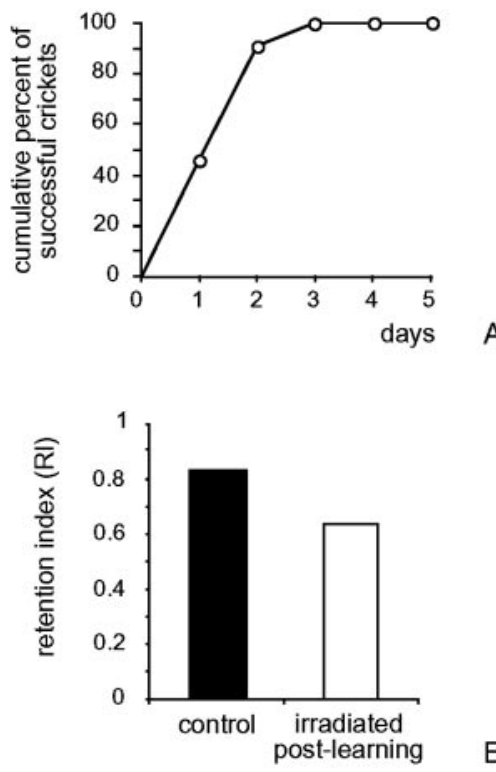

\section{B}

Figure 6. Effect of postlearning irradiation on retention performances. Irradiation was performed $6 \mathrm{hr}$ after the last learning session; retention performances were tested $24 \mathrm{hr}$ later. $A$, Learning performances of untreated crickets provided with olfactory cues $(n=11)$. B, Retention performances $1 \mathrm{~d}$ after irradiation. The RI of these crickets irradiated after learning is compared here with the Rl of the control crickets of Figure $5 D$. No significant difference is observed between the two groups.

thermore, locomotion and ovarian maturation of irradiated females were similar to those of control females. The efficiency of irradiation for neuroblast destruction together with the absence of side effects validated its use for subsequent behavioral analysis.

Mechanical, chemical, and genetic manipulations have demonstrated the involvement of mushroom bodies in learning and memory processes (for review, see Erber et al., 1987; Davis, 1996). We thus hypothesized that newly generated Kenyon cells might play a role in learning tasks. Proboscis extension reflex (PER) conditioning and electric conditioning are commonly used, respectively, in honeybees and Drosophila (Takeda, 1961; Quinn et al., 1974; Bitterman et al., 1983). In both species, mushroom body neuroblasts degenerate before adulthood (Ito and Hotta, 1992; Farris et al., 1999; Ganeshina et al., 2000). Therefore, because adult neurogenesis is not found in these species, the cricket is currently one of the best invertebrate model species for studying the functional role of newly generated neurons in the adult animal. The learning tests used in flies and bees were not easily transposable to the house cricket. We first tried the Tennessee Williams paradigm used for the cockroach (Mizunami et al., 1993, 1998), itself adapted from the water maze paradigm used in rodents and consisting of one unheated platform hidden in a heated surface. Unfortunately, it did not work because the first motivation of crickets was to escape from the closed arena. This escape behavior was repeatedly observed in different types of tests we tried. This led us to develop a new learning paradigm based on the natural tendency of the house cricket to escape from a closed box, which we named the escape paradigm. The insect was asked to choose between two holes, one allowing it to escape and the other one leading to a trap. Either olfactory or visual conditioning can be achieved. To minimize the possibility of success by luck, we considered that crickets had learned the task only if they made zero $(p=0.004)$ or one mistake $(p=0.035)$ in eight consecutive trials. Whatever the sensory mode used for conditioning (visual or olfactory cues), control crickets appeared to learn the task in a few days because after five learning sessions, almost all of them were successful. These results validate this new operant conditioning for our insect model. The retention index indicated that, whereas $90 \%$ of control crickets were able to remember the task $24 \mathrm{hr}$ after acquisition when olfactory cues were used, they forgot the task very quickly when visual cues alone were provided. These data suggest that the visual cues that we provided failed to induce strong memory of the conditioning. We do not exclude that other visual patterns might be more efficient to establish stable memory. It is noteworthy that crickets are nocturnal insects that rely on olfaction for foraging, and visual information might not be particularly relevant for them. Seven days later, control crickets failed to remember the task, whatever the sensory mode used. By contrast, in another cricket (Gryllus bimaculatus), the retention of elementary olfactory conditioning has been shown to last up to 10 weeks (Matsumoto and Mizunami, 2002). In contrast to the honeybee and Drosophila, in which specific molecular mechanisms underlying short-, medium-, and long-term memory have been identified, nothing is known about the different phases of memory in Gryllidae. Because the escape paradigm is developed over several days, the kinetics are completely different from those involved in the PER conditioning used to study memory phases. It is therefore hazardous to establish parallels between these two models.

In contrast to control animals, learning performance of irradiated crickets depended on the type of sensory cues used. When olfactory cues alone were provided, learning was significantly retarded compared with that of control crickets, and retention was strongly reduced. These learning and memory deficiencies were unlikely to be attributable to altered olfaction ability of irradiated crickets because they still presented a clear preference for vanilla over orange, as control crickets did. When visual cues alone were provided, irradiated crickets exhibited slightly slower learning, but it was not significantly different from that of controls, suggesting that visual abilities were not highly affected by irradiation. When both sensory cues were concomitantly supplied, acquisition speed and the success rate of irradiated crickets were identical to those of control animals. Interestingly, whereas acquisition was improved by the association of visual and olfactory cues, retention performances of irradiated insects seemed to be impaired by this association. This suggests that different processes and neural networks are involved during acquisition and memory storage, because it has been shown that different molecular mechanisms underlie short-, medium-, and long term memory (for review, see Menzel, 1999, 2001). The fact that irradiated crickets learned as well as control insects when both cues were provided allows us to rule out the hypothesis that altered performances could be ascribed to nonspecific deleterious effects of irradiation. In addition, to definitely rule out unspecific irradiation effects, we performed a control experiment in which intact crickets were allowed to learn the olfactory task and were then irradiated between the end of acquisition and the retention test. Because these animals exhibited normal retention scores, it seems that irradiation per se did not affect neuronal transmission.

In all our behavioral analyses, training sessions started 1 week after irradiation and lasted for up to $5 \mathrm{~d}$; consequently, retention tests were performed up to $19 \mathrm{~d}$ after irradiation. Thus, irradiated crickets lacked all the neurons that should have been generated during this period. Previous immunohistological studies combining BrdU and lachesin labeling (a marker of insect immature neurons) showed that newborn Kenyon cells begin to extend neurites into the peduncle a few days after their birth and project into specific areas of the pedunculus and of the lobes (Malaterre 
et al., 2002). Our results clearly show that adult-born neurons are not essential for learning but that they do influence learning kinetics. Reasonable assumptions can be made about immature neurons compared with mature neurons: because they are structurally plastic, they are highly susceptible to environmental changes and to different life experiences (Gould and Gross 2002). In cricket mushroom bodies, immunostaining and electron microscopic studies showed that the subpopulation of immature Kenyon cells expressed different neurotransmitters and presented specific arborization patterns (Strambi et al., 1998; Schürmann et al., 2000; Malaterre et al., 2002). Thus, newly born Kenyon cells probably present higher plasticity in terms of synaptogenesis and modulation of synaptic strength in response to environmental cues. In adult rodents, newborn hippocampal neurons display more robust long-term potentiation than mature interneurons (Wang et al., 2000). Thus, the continual influx of neurons that are temporarily immature (rather than a total increase in neuron number) could underlie behavioral abilities (Gould and Gross, 2002). We suggest that these immature neurons could increase the acquisition rate in this complex olfactory operant paradigm.

We cannot totally rule out the possibility that the behavioral effects observed could be the consequence of the diminution of the total mushroom body neuron number rather than of the absence of newly generated neurons. However, this hypothesis seems unlikely. Numerous studies emphasize that the mushroom body cortex is not constituted of a homogenous group of interneurons but is composed of different populations of Kenyon cells, characterized by the expression of different transcription factors and signaling proteins (Yang et al., 1995; Crittenden et al., 1998; Sinakevitch et al., 2001). Such mushroom body subdivisions are highly suggestive of different functional roles for these interneurons.

Using hydroxyurea during larval development, Malun et al. (2002) obtained honeybees with partial mushroom body ablation and demonstrated that it did not affect acquisition and retention of PER conditioning. These authors concluded that mushroom body integrity was not necessary for elementary olfactory discrimination tasks, for which antennal lobes might be sufficient. In contrast to the PER conditioning, our olfactory conditioning does not rely on a simple reflex but is a more complex conditioning in which the cricket has to make a choice. Mushroom bodies might be involved in complex learning tasks but not in simple elementary tasks, as supported by the work of Liu et al. (1999), showing that mushroom bodies are not required for visual learning in Drosophila but are needed for context generalization in visual conditioning. In future studies, it would therefore be interesting to investigate whether irradiated cricket performance would be impaired in more complex visual conditioning (e.g., with more than two opened holes) or whether the sensory modality used for conditioning is really a determinant in crickets lacking adult neurogenesis, as suggested by the present results.

\section{References}

Abercrombie M (1946) Estimation of nuclear population from microtome sections. Anat Rec 94:239-247.

Barnea A, Nottebohm F (1996) Recruitment and replacement of hippocampal neurons in young and adult chickadees: an addition to the theory of hippocampal learning. Proc Natl Acad Sci USA 93:714-718.

Bayer SA, Yakel JW, Puri PS (1982) Neurons in the rat dentate gyrus granular layer substantially increase during juvenile and adult life. Science 216:890-892.

Bitterman ME, Menzel R, Fietz A, Schäfer S (1983) Classical conditioning of proboscis extension in honeybees (Apis mellifera). J Comp Psychol 97:107-119.

Cayre M, Strambi C, Strambi A (1994) Neurogenesis in an adult insect brain and its hormonal control. Nature 368:57-59.

Cayre M, Strambi C, Charpin P, Augier R, Meyer MR, Edwards JS, Strambi A (1996a) Neurogenesis in adult insect mushroom bodies. J Comp Neurol 371:300-310.

Cayre M, Strambi C, Charpin P, Augier R, Renucci M, Strambi A (1996b) Inhibition of polyamine biosynthesis alters oviposition behavior in female crickets. Behav Neurosci 110:1117-1125.

Cayre M, Strambi C, Charpin P, Augier R, Strambi A (1997) Inhibitory role of ecdysone on neurogenesis and polyamine metabolism in the adult cricket brain. Arch Insect Biochem Physiol 35:85-97.

Corotto FS, Henegar JA, Maruniak JA (1993) Neurogenesis persists in the subependymal layer of the adult mouse brain. Neurosci Lett 149:111-114.

Crittenden JR, Skoulakis EMC, Han KA, Kalderon D, Davis RL (1998) Tripartite mushroom body architecture revealed by antigenic markers. Learn Mem 5:38-51.

Davis RL (1996) Physiology and biochemistry of Drosophila learning mutants. Physiol Rev 76:299-317.

de Belle JS, Heisenberg M (1994) Associative odor learning in Drosophila abolished by chemical ablation of mushroom bodies. Science 263:692-695.

Erber J, Homberg U, Gronenberg W (1987) Functional roles of the mushroom bodies in insects. In: Arthropod brain (Gupta AP, ed), pp 485-512. New York: Wiley.

Eriksson PS, Perfilieva E, Björk-Eriksson T, Alborn AM, Nordborg C, Peterson DA, Gage FH (1998) Neurogenesis in the adult human hippocampus. Nat Med 4:1313-1317.

Farris SM, Robinson GE, Davis RL, Fahrbach SE (1999) Larval and pupal development of the mushroom bodies in the honey bee, Apis mellifera. J Comp Neurol 414:97-113.

Feng R, Rampon C, Tang YP, Shrom D, Jin J, Kyin M, Sopher B, Martin GM, Kim SH, Langdon RB, Sisodia SS, Tsien JZ (2001) Deficient neurogenesis in forebrain-specific presinilin-1 knockout mice is associated with reduced clearance of hippocampal memory traces. Neuron 32:911-926.

Ganeshina O, Schäfer S, Malun D (2000) Proliferation and programmed cell death of neuronal precursors in the mushroom bodies of the honey bee. J Comp Neurol 417:349-365.

Gould E, Gross CG (2002) Neurogenesis in adult mammals: some progress and problems J Neurosci 22:619-623.

Gould E, McEwen BS, Tanapat P, Galea LAM, Fuchs E (1997) Neurogenesis in the dentate gyrus of the adult tree shrew is regulated by psychosocial stress and NMDA receptor activation. J Neurosci 17:2492-2498.

Gould E, Tanapat P, McEwen BS, Flügge G, Fuchs E (1998) Proliferation of granule cell precursors in the dentate gyrus of adult monkeys is diminished by stress. Proc Natl Acad Sci USA 95:3168-3171.

Gould E, Beylin A, Tanapat P, Reeves A, Shors TJ (1999a) Learning enhances adult neurogenesis in the hippocampal formation. Nat Neurosci 2:260-265.

Gould E, Reeves AJ, Graziano MS, Gross CG (1999b) Neurogenesis in the neocortex of adult primates. Science 286:548-552.

Gronenberg W (2001) Subdivisions of hymenopteran mushroom body calyces by their afferent supply. J Comp Neurol 435:474-489.

Gu SH, Tsia WH, Chiang AS, Chow YS (1999) Mitogenic effects of 20hydroxyecdysone on neurogenesis in adult mushroom bodies of the cockroach, Diploptera punctata. J Neurobiol 39:264-274.

Hammer M, Menzel R (1995) Learning and memory in the honeybee. J Neurosci 15:1617-1630.

Heisenberg M (1994) Central brain function in insects: genetic studies on the mushroom bodies and central complex in Drosophila. In: Fortschritte der Zoologie: neural basis of behavioral adaptations (Rathmayer W, ed), pp 61-79. Stuttgart: Fisher.

Heisenberg M (1998) What do the mushroom bodies do for the insect brain? An introduction. Learn Mem 5:1-10.

Ito K, Hotta Y (1992) Proliferation pattern of postembryonic neuroblasts in the brain of Drosophila melanogaster. Dev Biol 149:25-34.

Kempermann G, Kuhn HG, Gage FH (1997) More hippocampal neurons in adult mice living in an enriched environment. Nature 386:493-495.

Krugers HJ, Douma BR, Andringa G, Bohus B, Korf J, Luiten PG (1997) Exposure to chronic psychosocial stress and corticosterone in the rat: 
effects on spatial discrimination learning and hippocampal protein kinase $\mathrm{C} \gamma$ immunoreactivity. Hippocampus 7:427-436.

Lemaire V, Koehl M, Le Moal M, Abrous DN (2000) Prenatal stress produces learning deficits associated with an inhibition of neurogenesis in the hippocampus. Proc Natl Acad Sci USA 97:11032-11037.

Li XC, Jarvis ED, Alvarez-Borda B, Lim DA, Nottebohm F (2000) A relationship between behavior, neurotrophin expression, and new neuron survival. Proc Natl Acad Sci USA 97:8584-8589.

Liu L, Wolf R, Ernst R, Heisenberg M (1999) Context generalization in Drosophila visual learning requires the mushroom bodies. Nature 400:753-756.

Lois C, Alvarez-Buylla A (1994) Long-distance neuronal migration in the adult mammalian brain. Science 264:1145-1148.

Malaterre J, Strambi C, Chiang AS, Aouane A, Strambi A, Cayre M (2002) Development of cricket mushroom bodies. J Comp Neurol 452:215-227.

Malun D (1998) Early development of mushroom bodies in the brain of the honeybee Apis mellifera as revealed by BrdU incorporation and ablation experiments. Learn Mem 5:90-101.

Malun D, Plath N, Giurfa M, Moseleit AD, Müller U (2002) Hydroxyureainduced partial mushroom body ablation in the honeybee Apis mellifera: volumetric analysis and quantitative protein determination. J Neurobiol 50:31-44.

Matsumoto Y, Mizunami M (2000) Olfactory learning in the cricket Gryllus bimaculatus. J Exp Biol 203:2581-2588.

Matsumoto Y, Mizunami M (2002) Lifetime olfactory memory in the cricket Gryllus bimaculatus. J Comp Physiol [A] 188:295-299.

Menzel R (1999) Memory dynamics in the honeybee. J Comp Physiol [A] $185: 323-340$

Menzel R (2001) Searching for the memory trace in a mini-brain, the honeybee. Learn Mem 8:53-62.

Mizunami M, Weibrecht JM, Strausfeld NJ (1993) A new role for the insect mushroom bodies: place memory and motor control. In: Biological neural networks in invertebrate neuroethology and robotics (Beer RD, ed), pp 199-225. Cambridge, UK: Academic.

Mizunami M, Weibrecht JM, Strausfeld NJ (1998) Mushroom bodies of the cockroach: their participation in place memory. J Comp Neurol 402:520-537.

Mobbs PG (1982) The brain of the honeybee Apis mellifera. I. The connections and spatial organization of the mushroom bodies. Philos Trans $\mathrm{R}$ Soc B Biol Sci 298:309-394.

Mobbs PG (1984) Neural networks in the mushroom bodies of the honeybee. J Insect Physiol 30:43-58.

Nilsson M, Perfilieva E, Johansson U, Orwar O, Eriksson PS (1999) Enriched environment increases neurogenesis in the adult rat dentate gyrus and improves spatial memory. J Neurobiol 39:569-578.

Oland LA, Tolbert LP, Mossman KL (1988) Radiation-induced reduction of the glial population during development disrupts the formation of olfactory glomeruli in an insect. J Neurosci 8:353-367.

Peißner W, Kocher M, Treuer H, Gillardon F (1999) Ionizing radiationinduced apoptosis of proliferating stem cells in the dentate gyrus of the adult rat hippocampus. Mol Brain Res 71:61-68.
Quinn WG, Harris WA, Benzer S (1974) Conditioned behavior in Drosoph ila melanogaster. Proc Natl Acad Sci USA 71:708-712.

Rage P, Renucci M (1985) Saisie de données dans l'étude du comportement animal. Electron Applic 44:87-92.

Renucci M, Cherkaoui L, Rage P, Augier R, Strambi A (1992) Juvenile hormone exerts a primer effect on oviposition behavior in Acheta domesticus. In: Insect juvenile hormone research: fundamental and applied approaches (Mauchamp B, Couillaud F, Baehr JC, eds), pp 147-163. Paris: Institut National de Recherche Agronomique Editions.

Rochefort C, Gheusi G, Vincent JD, Lledo PM (2002) Enriched odor exposure increases the number of newborn neurons in the adult olfactory bulb and improves odor memory. J Neurosci 22:2679-2689.

Scharff C, Kirn JR, Grossman M, Macklis JD, Nottebohm F (2000) Targeted neuronal death affects neuronal replacement and vocal behavior in adult songbirds. Neuron 25:481-492.

Schürmann FW, Ottersen OP, Honegger HW (2000) Glutamate-like immunoreactivity marks compartments of the mushroom bodies in the brain of the cricket. J Comp Neurol 418:227-239.

Scotto-Lomassese S, Strambi C, Strambi A, Charpin P, Augier R, Aouane A, Cayre M (2000) Influence of environmental stimulation on neurogenesis in the adult insect brain. J Neurobiol 45:162-171.

Scotto-Lomassese S, Strambi C, Aouane A, Strambi A, Cayre M (2002) Sensory inputs stimulate progenitor cell proliferation in an adult insect brain. Curr Biol 12:1001-1005.

Shors TJ, Miesegaes G, Beylin A, Zhao M, Rydel T, Gould E (2001) Neurogenesis in the adult is involved in the formation of trace memories. Nature 410:372-375.

Sinakevitch I, Farris SM, Strausfeld NJ (2001) Taurine-, aspartate- and glutamate-like immunoreactivity identifies chemically distinct subdivisions of Kenyon cells in the cockroach mushroom body. J Comp Neurol 439:352-367.

Strambi C, Cayre M, Sattelle DB, Augier R, Charpin P, Strambi A (1998) Immunocytochemical mapping of an RDL-like GABA receptor subunit and of GABA in brain structures related to learning and memory in the cricket, Acheta domesticus. Learn Mem 5:78-89.

Tada E, Yang C, Gobbel GT, Lamborn R, Fike JR (1999) Long-term impairment of subependymal repopulation following damage by ionizing irradiation. Exp Neurol 160:66-77.

Tada E, Parent JM, Lowenstein DH, Fike JR (2000) X-irradiation causes a prolonged reduction in cell proliferation in the dentate gyrus of adult rats. Neuroscience 99:33-41.

Takeda K (1961) Classical conditioned response in the honeybee. J Insect Physiol 6:168-179.

van Praag H, Kempermann G, Gage FH (1999) Running increases cell proliferation and neurogenesis in the adult mouse dentate gyrus. Nat Neurosci 2:266-270.

Wang S, Scott BW, Wojtowicz JM (2000) Heterogeneous properties of dentate granule neurons in the adult rat. J Neurobiol 42:248-257.

Yang MY, Armstrong JD, Vilinsky I, Strausfeld NJ, Kaiser K (1995) Subdivision of the Drosophila mushroom bodies by enhancer-trap expression patterns. Neuron 15:45-54. 